\title{
LA PSICOLOGÍA EN AMÉRICA LATINA: ENTRE LA BÚSQUEDA DE IDENTIDAD Y EL ESFUERZO POR RECONOCIMIENTO GLOBAL ${ }^{\text {do }}$
}

\author{
Andrés M. Pérez-Acosta ${ }^{\mathrm{a} 1} \mathbb{D}, \boldsymbol{\&}$ Andrés J. Consoli $^{\mathrm{b}}$ (i) \\ Universidad del Rosario, Bogotá, Colombia. ${ }^{\text {a }}$ \\ University of California, Santa Barbara, United States of America. ${ }^{\mathrm{b}}$
}

\section{RESEÑA DEL LIBRO}

Ardila, R. (Ed.) (2018). Psychology in Latin America: Current status, challenges and perspectives. Cham, Suiza: Springer Nature. https://doi.org/10.1007/978-3-319-93569-0

The isolation that characterized psychology in this part of the world at the turn of the century has become effete in a shared and instantly connected world where language, national, and regional barriers are quickly being positively eroded.

Saths Cooper (2018)

En 1986, el Dr. Rubén Ardila publicó el libro La Psicología en América Latina, Pasado, Presente y Futuro, en la editorial Siglo XXI de México. Como muchas de las obras de Ardila, aquella fue otro trabajo pionero en su tema. Justamente aquel panorama de la psicología en nuestro continente es el principal antecedente de la compilación Psychology in Latin America: Current status, challenges and perspectives (La Psicología en América Latina: Estado Actual, Desafíos y Perspectivas), compilada también por Ardila y publicada por la prestigiosa editorial Springer (Ardila, 2018).

El libro de 1986, escrito y publicado en español, se dirigió especialmente a los psicólogos de la región, con la intención de ofrecer un contexto histórico y cultural, además de un panorama de las investigaciones y las aplicaciones profesionales, mientras señalaba las limitaciones y los caminos por recorrer. El eje común a todos aquellos aspectos fue la búsqueda de identidad. Treinta y dos años después, 21 psicólogos latinoamericanos unieron esfuerzos para ofrecer un panorama renovado. La cuestión de

${ }^{1}$ Correspondence about this article should be addressed to Andrés M. Pérez-Acosta: andres.perez@urosario.edu.co 
la identidad sigue presente en esta nueva obra, pero también se nota de manera sustantiva la búsqueda de un reconocimiento global de la psicología en Latinoamérica, dada su madurez. Para alcanzar este objetivo, la obra ha sido compilada en inglés y está dirigida a toda la comunidad internacional de psicólogos.

Difícilmente otro compilador podría ser más pertinente. Entre los logros más significativos Ardila y su influencia en la psicología generada en América Latina cabe destacar haber sido el fundador de la Revista Latinoamericana de Psicología hace 50 años (1969; véase Salas et al., 2019). Esta publicación periódica se convirtió rápidamente en el principal foro de la psicología como ciencia y profesión en América Latina. En un contexto acostumbrado a publicaciones que no llegan a sostenerse en el tiempo por varias y diversas razones, la edición ininterrumpida de la Revista por más de medio siglo es un hito entre las publicaciones científicas de la región. Ardila mismo se define como un psicólogo latinoamericano con compromiso social quien se ha dedicado al fortalecimiento internacional de la psicología en múltiples frentes: investigación, docencia, agremiación, entre otros (véase Consoli et al., 2017).

El autor del Prólogo es Saths Cooper, de Sudáfrica, actual Past President de la Unión Internacional de Ciencia Psicológica, conocida como IUPsyS, por sus iniciales en inglés. Cooper ofrece al mismo tiempo una mirada a la distancia y empática acerca de la llamativa paradoja de un gran desarrollo científico y profesional de la psicología en Latinoamérica, junto con un aislamiento que persiste por dominancias hegemónicas tanto lingüísticas como culturales y geográficas en la psicología. No obstante, él también se muestra optimista sobre el futuro, precisamente gracias a esta compilación de once capítulos (consultar epígrafe).

Los lectores encontrarán de máxima utilidad el Prefacio escrito por el compilador. Allí Ardila sintetiza las principales ideas presentadas en el libro. Seguido al prefacio se presentan las contribuciones de trece psicólogos y siete psicólogas de Argentina, Brasil, Chile, Colombia, México, Perú y Venezuela. Al respecto, nos anticipamos a las posibles críticas que siempre surgen hacia iniciativas de esta clase, relacionadas con representatividad de género y geográfica. Cualquier obra de esta magnitud, con el enorme trabajo editorial que supone, no pretende ni puede ser una muestra estadísticamente significativa ni políticamente pre-establecida. Lo que está claro es que los veinte autores, como unidad, constituyen un conjunto de destacados y destacadas colegas, expertos y expertas en sus respectivos campos disciplinares y profesionales, con reconocimiento tanto en sus países como en la región. En conjunto, los científicos y profesionales 
reunidos por la encomiable capacidad de convocatoria de Ardila dada su reputación como referente de la región permite al lector un acceso pleno e inusual al pulso de la psicología en América Latina.

Del primer capítulo, Panorama of Psychology in Latin America, destacamos la interesante lista que Ardila propone como soluciones para el histórico problema del aislamiento de la psicología de la región:

1. Organizar amplios programas internacionales de intercambio de estudiantes y profesores.

2. Crear redes internacionales de investigadores que aprovechen las facilidades derivadas de Internet.

3. Promover la participación de psicólogos latinoamericanos en los principales eventos globales de la disciplina.

4. Enfatizar en el aprendizaje del inglés, el cual se ha convertido en el lenguaje de la ciencia, incluida la psicología.

5. Organizar grandes eventos internacionales de la psicología en América Latina (de hecho, el Congreso Internacional de Psicología de 2024 se efectuará en Río de Janeiro).

6. Involucrarse en proyectos que tengan sede en centros mundialmente reconocidos de investigación y desarrollo, con miras a la generación de futuros centros, programas y proyectos regionales de alta calidad.

En el segundo capítulo, los Dres. Germán Gutiérrez (Colombia) y Jesús LandeiraFernández (Brasil) ofrecen una completa y profunda perspectiva actual y proyecciones de la investigación psicológica en Latinoamérica. Los autores articulan las principales tendencias en investigación básica y aplicada, los métodos más usuales y los avances y limitaciones derivados de sus resultados. Lanzan una clara petición de fortalecimiento de los programas de investigación, locales, regionales e internacionales, lo cual supone un necesario aumento en los presupuestos de financiación, institucionales y nacionales.

También con gran detalle y documentación, los historiadores de la psicología Dres. Hugo Klappenbach, Sebastián Vásquez-Ferrero y Miguel Gallegos (Argentina) relatan en el capítulo tres el largo camino conducente a la profesionalización de la psicología en América Latina. Ellos describen y analizan el entrenamiento de los psicólogos en diferentes países, sus fundamentos conceptuales, las necesidades sociales y desarrollos de la disciplina que llevaron a la creación de las carreras de psicología, la inserción de la profesión en la sociedad y otros asuntos relacionados. 
El Dr. Héctor Fernández-Álvarez y la Dra. Claudia Bregman (Argentina) presentan la perspectiva latinoamericana de la psicología clínica y de la salud, en el capítulo cuatro. En el caso particular de la psicología clínica, es el área con el más grande número de profesionales en la región, haciendo eco de la tendencia norteamericana y europea. Por su parte, la psicología de la salud se ha desarrollado de manera muy importante en la región en las últimas décadas. Fernández-Álvarez y Bregman muestran la evolución de estos campos desde comienzos del siglo $\mathrm{XX}$, durante el cual se ha enriquecido el propósito inicial de la evaluación y el diagnóstico, con el desarrollo de las intervenciones terapéuticas, tanto en clínica como en salud, eso sí con enormes divergencias epistemológicas y metodológicas aún vigentes.

La psicología del desarrollo en América Latina es abordada en el capítulo cinco por las Dras. Silvia H. Koller y Normanda Araujo De Morais (Brasil). Las autoras hacen énfasis en la evolución del campo del desarrollo humano en las últimas tres décadas, en tres partes: en la primera ofrecen una perspectiva de la región; en la segunda presentan avances en algunos países selectos (Argentina, Chile, Colombia, Paraguay, Perú, Uruguay y Venezuela); y en la tercera se enfocan en los desarrollos de su país, Brasil. Finalmente, Koller y De Morais plantean futuras direcciones de investigación y práctica en esta importante área.

El capítulo seis se titula Educational and School Psychology in Latin American Countries: Challenges and New Possibilities (Psicología Educativa y Escolar en América Latina: Desafios y Nuevas Posibilidades). Sus autores, la Dra. Maria Regina Maluf y el Dr. Renan de Almeida Sargiani (Brasil), parten de la estrecha relación entre la psicología del desarrollo y la psicología educativa y escolar. Presentan el contexto del surgimiento de este campo dentro de la educación, tanto en Europa como en Estados Unidos. Finalmente, Maluf y Sargiani sintetizan los más importantes desarrollos latinoamericanos y las perspectivas de este campo en la región.

Una completa colaboración internacional es el capítulo siete, que muestra a la psicología del trabajo y organizacional en Latinoamérica. Sus autores, los Dres. Jairo Eduardo Borges-Andrade (Brasil), Érico Rentería Pérez (Colombia) y Juan Pablo Toro (Chile), se enfrentaron al reto de sintetizar lo que bien denominan una "amalgama" de valores, aspectos organizacionales, asuntos legales, bloques económicos, lenguajes y personas de América Latina. Luego de revisar 110 fuentes sobre 14 países, organizaron su capítulo en los siguientes aspectos: 1. Campos de acción; 2. Historia; 3. La práctica profesional; 4. La enseñanza; 5 . La producción y diseminación de conocimiento en la 
psicología del trabajo y organizacional latinoamericana. A su vez, dividieron cada sección según los desarrollos particulares de varios países. Finalmente, el capítulo cierra con unas detalladas Conclusiones acerca de un campo complejo y en rápida evolución.

Psychological, Sociological, and Cultural Social Psychology in Latin America, es el título del capítulo ocho, a cargo del Dr. Rolando Díaz-Loving y la Dra. Cinthia Cruz del Castillo (México). Como se aprecia en el título, ya es evidente la vigente división de enfoques de la psicología social latinoamericana entre las vertientes psicológica, sociológica y cultural. Con todo, ha sido América Latina un continente muy influyente a nivel global, a diferencia de lo que sucede con otras áreas tradicionales de la psicología. El aporte de eminentes psicólogos como los Dres. Rogelio Díaz-Guerrero e Ignacio Martín-Baró ha traspasado los límites nacionales y regionales, convirtiéndose en señales de identidad de la psicología latinoamericana, con los epónimos de la etnopsicología y la psicología de la liberación, respectivamente.

A propósito, la destacada psicóloga venezolana Maritza Montero plantea en el capítulo nueve la psicología comunitaria como una creación latinoamericana, justamente de la mano de los esfuerzos de Ignacio Martín-Baró. La Dra. Montero introduce con el “amanecer" de la psicología comunitaria en América Latina (1975-1989). Posteriormente relata la construcción de las bases de la psicología comunitaria (1990-1999). Luego diferencia lo que es la "comunidad" y lo que es la "psicología comunitaria". A continuación, presenta secciones de corte metodológico, conceptual y ético. Finalmente, cierra con cuatro ejemplos actuales de la psicología comunitaria latinoamericana, que se están desarrollando en Guatemala, México, Venezuela y Brasil.

La psicóloga colombiana Ángela Tapias presenta en el capítulo diez la psicología jurídica y forense en Latinoamérica. Tapias es reconocida en este campo por ser la fundadora de la Asociación Latinoamericana de Psicología Jurídica y Forense. A pesar de que este campo se originó en los inicios del siglo XX, su desarrollo en nuestra región es relativamente reciente. Tapias comienza el capítulo con una reseña histórica de la introducción del campo en América Latina, enfatizando cómo la psicología se ha introducido en los sistemas legales de los diferentes países. Luego presenta los programas de posgrado en psicología jurídica y forense en Centro y Sudamérica. A continuación presenta las asociaciones profesionales, los líderes e hitos por países. Concluye, por último, con un campo en pleno desarrollo, promisorio pero que requiere un fortalecimiento principalmente en la formación especializada. 
El capítulo final, sobre tendencias actuales y perspectivas, tiene nombre y sello propio: el Dr. Reynaldo Alarcón, el principal psicólogo peruano y uno de los grandes psicólogos latinoamericanos, que siempre ha tenido la capacidad de visión panorámica de la psicología en la región, tanto en su faceta histórica como en la faceta prospectiva (véase Alarcón, 2002). Alarcón mezcla adecuadamente el análisis histórico y conceptual, con el fin de mostrar la evolución de la psicología en América Latina, que ha pasado de ser una novedad traída por un puñado de migrantes europeos a ser un campo de aportes propios e influyentes, de vuelta hacia Estados Unidos y Europa.

En síntesis, Psychology in Latin American: Current status, challenges and perspectives es una obra de consulta obligatoria para aquellas personas interesadas en la historia, estado actual y perspectivas de la psicología en América Latina. En ese sentido, sería útil en los niveles de pregrado y posgrado de psicología, especialmente en campos como historia de la psicología, psicología organizada y psicología internacional, además de las áreas introducidas en los capítulos reseñados (psicología del desarrollo, educativa y escolar, clínica, de la salud, del trabajo y organizacional, social, comunitaria, jurídica y forense).

Las personas interesadas pueden consultar más datos en la página de acceso al libro, la cual ofrece diferentes formatos, tanto en papel como electrónicos: www.springer.com/gp/book/9783319935683. 


\section{Referencias}

Alarcón, R. (2002). Estudios sobre psicología latinoamericana. Lima: Universidad Ricardo Palma.

Ardila, R. (1986). La psicología en América Latina, pasado, presente y futuro. México: Siglo XXI.

Ardila, R. (Ed.) (2018). Psychology in Latin America: Current status, challenges and perspectives. Cham, Suiza: Springer Nature. https://doi.org/10.1007/978-3-31993569-0

Consoli, A. J., Pérez-Acosta, A. M., Morgan-Consoli, M. L., Ramiro Sánchez, T., Romero Morales, A., \& Vanegas Martínez, G. (2017). International organized psychology and social commitment: Rubén Ardila - Colombia. International Psychology Bulletin, 21(3), 66-72.

Cooper, S. (2018). Foreword. En: R. Ardila (Ed.), Psychology in Latin America: Current status, challenges and perspectives (pp. v-vi). Cham, Suiza: Springer Nature. https://doi.org/10.1007/978-3-319-93569-0

Salas, G., Vega-Arce, M., González, C., Ossa, J. C., Cudina, J. N., Caycho-Rodríguez, T., Barboza-Palomino, M., Ventura-León, J., Guerra-Labbé, L. \& López-López, W. (2019). The fiftieth anniversary of the Revista Latinoamericana de Psicología: A bibliometric analysis. Revista Latinoamericana de Psicología, 51(2), 206-218. 